\title{
Adelieledone, a new genus of octopodid from the Southern Ocean
}

\author{
A.L. ALLCOCK ${ }^{1}$, F.G. HOCHBERG ${ }^{2}$, P.G.K. RODHOUSE ${ }^{3}$ and J.P. THORPE ${ }^{4}$ \\ ${ }^{1}$ School of Biology and Biochemistry, Queen's University Belfast, 97 Lisburn Road, Belfast BT9 7BL, UK \\ l.allcock@qub.ac.uk \\ ${ }^{2}$ Department of Invertebrate Zoology, Santa Barbara Museum of Natural History, 2559 Puesta del Sol Road, Santa Barbara, \\ CA 93105, USA \\ ${ }^{3}$ British Antarctic Survey, NERC, High Cross, Madingley Road, Cambridge CB3 OET, UK \\ ${ }^{4}$ Department of Ecology and Evolutionary Biology, Liverpool University, Port Erin Marine Laboratory, Port Erin, \\ Isle of Man IM9 6JA, UK
}

\begin{abstract}
The syntypes of the endemic Southern Ocean octopodid Pareledone polymorpha (Robson, 1930) were re-examined and measurements, counts and indices are presented. The two forms described by Robson, namely oblonga and affinis, are determined to have no taxonomic validity. The species polymorpha shows morphological similarities with Pareledone adelieana (Berry, 1917) but differs in relative arm lengths, sucker counts, external colouration and size at maturity. Both species are transferred to the new genus Adelieledone, which is separated from the genus Pareledone s.s. by the transverse ridges in the ligula groove of the hectocotylus, the sharp tip of the lower beak, the enlarged posterior salivary glands, the absence of stylets and by skin sculpture, especially by the presence of two longitudinal integumentary ridges on the dorsal mantle. A new species, Adelieledone piatkowski, is described from the Antarctic Peninsula. Beak morphology can discriminate the genera in predator studies.
\end{abstract}

Received 16 October 2002, accepted 2 June 2003

Key words: Antarctica, Cephalopoda, Octopodidae, Pareledone, taxonomy

\section{Introduction}

The endemic Southern Ocean octopod genus Pareledone is abundant and diverse (Allcock et al. 2001) and is of some ecological importance; its species feed on amphipods, polychaetes, fish and ophiuroids (Piatkowski et al. in press) and are fed on by top predators such as pinnipeds (Klages 1996) including southern elephant seals, Mirounga leonina L., 1758 (Rodhouse et al. 1992) and Weddell seals, Leptonychotes weddellii (Lesson, 1826) (Lipinski \& Woyciechowski 1981).

The wide morphological variability that exists within Pareledone s.l. has been noted in many studies and the status of the genus has often been questioned. Voight (1993) suggested there was no conclusive evidence that the congeners shared an evolutionary history. Daly \& Rodhouse (1994) illustrated wide morphological differences in the beaks and hectocotyli of Pareledone polymorpha (Robson, 1930) and P. turqueti (Joubin, 1905), whilst Ogden et al. (1998) commented further on the unusual beak of P. polymorpha. Lu \& Stranks (1994) cited potential morphological reasons, including the W-shaped funnel organ and ribbed ligula groove, for removing $P$. adelieana (Berry 1917) and its sister taxon P. polymorpha from Pareledone s.s. Recent genetic evidence (Carlini et al. 2001, Allcock \& Piertney 2002) suggests that Pareledone is not monophyletic and supports the morphological findings. These studies have led to a better understanding of species within the group and we are now able to define the characters that separate $P$. polymorpha and $P$. adelieana from Pareledone s.s. We have used this information to erect a new genus, Adelieledone, to accommodate these two species.

The removal of adelieana and polymorpha considerably simplifies the diagnosis of the genus Pareledone and may help remove some of the controversy that has traditionally surrounded this genus. We have included a revised diagnosis.

Robson (1932) suggested that there were two varieties of polymorpha: oblonga and affinis. Deformation associated with preservation is well documented and polymorpha is a gelatinous species that might be particularly prone to such deformation. However, while re-examining the type series of polymorpha, we realised it included several specimens of $P$. turqueti, removal of which considerably reduced the variability within the series. We include a redescription based on correctly identified material in the type series.

A third new species of Adelieledone has been discovered during the course of this work and is described herein.

Abbreviations and indices used throughout this paper follow Roper \& Voss (1983). Indices are given as minmean-max. Specimen repositories are abbreviated as: $\mathrm{BMNH}=$ Natural History Museum, London; NMSZ = National Museums of Scotland, Edinburgh; MV = Museum Victoria, Melbourne; AM = Australian Museum, Sydney; MNHN = Muséum National d'Histoire Naturelle, Paris. 


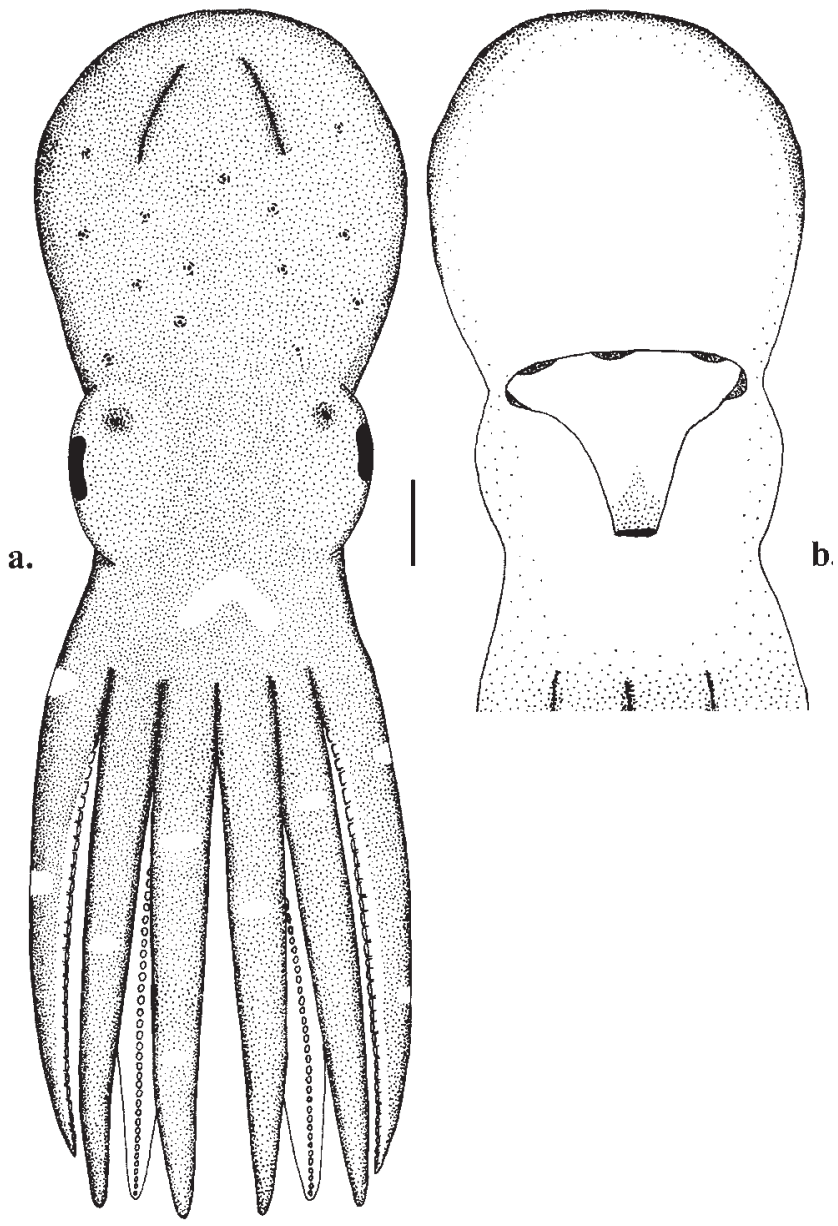

Fig. 1. External anatomy of Adelieledone polymorpha, NMSZ 2002037.029, 우, $57 \mathrm{~mm}$ ML. a. Dorsal view, b. Ventral view. Scale bar $10 \mathrm{~mm}$.

\section{Systematics \\ Octopodidae Orbigny, 1840 Adelieledone gen. nov.}

(Figs 1, 2a, 3a-e, 4)

Diagnosis: Benthic octopodids, ML to $90 \mathrm{~mm}$; mantle saccular, without fins; eight arms lacking cirri; arms with small (ASI 4-10) uniserial suckers, third right arm of males hectocotylized with end of arm clearly differentiated into ligula and calamus, ligula large (LLI 10-16), ligula groove long, well marked and deep with transverse ridges (Figs 3c $\& 4 c)$, arm tips not otherwise modified; web depth medium (WDI 20-40); funnel organ W-shaped (Fig. 3b); gills well developed, with 6-8 lamellae; ink sac present or reduced; crop well developed, posterior salivary glands large (length of posterior salivary glands up to twice the length of the buccal mass; Fig. 2a), chromatophores present within the connective tissue covering the dorsal surface of digestive gland; stylets absent; two short, longitudinal integumentary ridges on the mid-dorsal posterior mantle (Figs 1a \& 4a); diverticulum of penis not coiled (Fig. 3d), spermatophores medium length (SpLI 60-80) and slender; rostral tip of lower beak sharp (Fig. 3a); radula with nine elements (Fig. 3e), rachidian with large central cusp, rachidian may have other minor cusps.

Type species: Moschites adelieana Berry, 1917. By original designation.

Included species: Moschites adelieana Berry, 1917, Graneledone polymorpha Robson, 1930, Adelieledone piatkowski sp. nov.

Etymology: From the combination of Adélie (the type

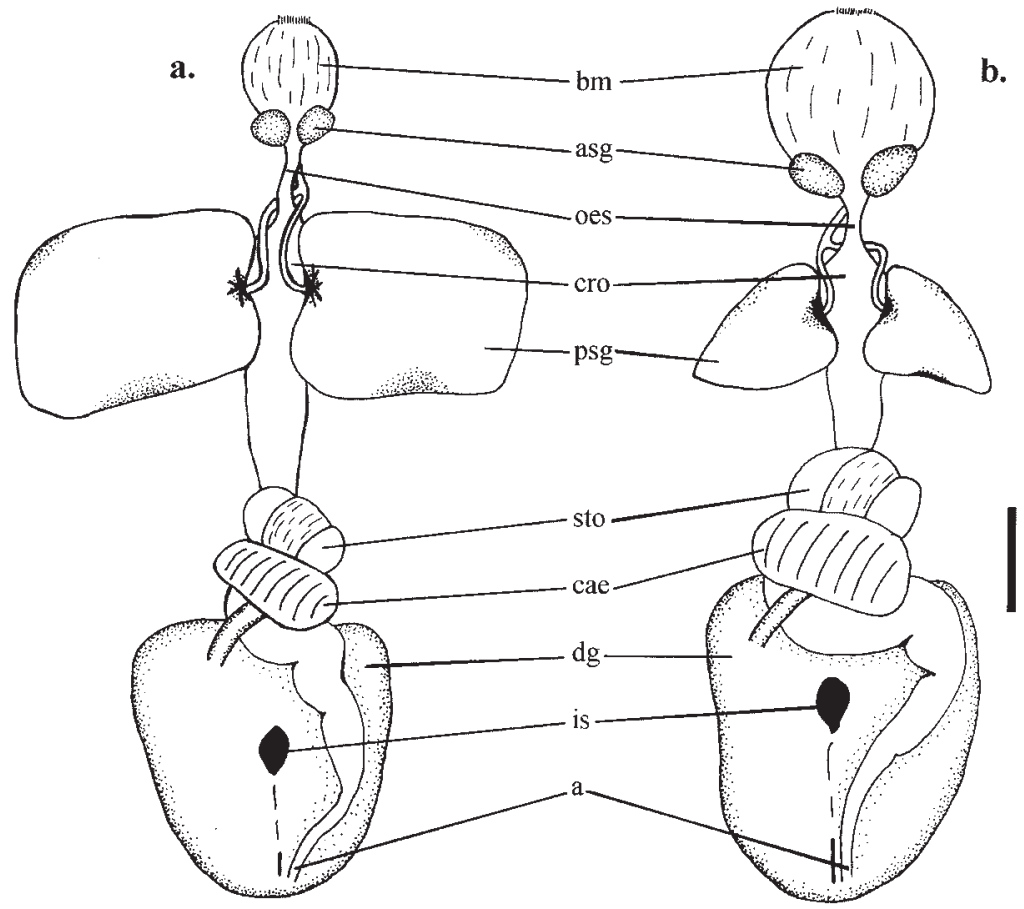

Fig. 2. Comparative anatomy of digestive systems. a. Adelieledone polymorpha, NMSZ 2002037.029, ণ', $75 \mathrm{~mm}$ ML, b. Pareledone turqueti, NMSZ 2002037.046, ণ̛, $78 \mathrm{~mm}$ ML. Scale bar $10 \mathrm{~mm}$. Abbreviations: $\mathrm{a}=$ anus, asg $=$ anterior salivary gland, $\mathrm{bm}=$ buccal mass, cae $=$ caecum, cro $=$ crop, $\mathrm{dg}=$ digestive gland, is = ink sac, oes = oesophagus, $\mathrm{psg}=$ posterior salivary gland, $\mathrm{sto}=$ stomach . 
a.
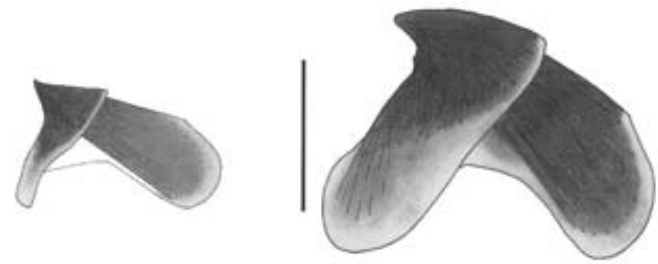

f.

b.

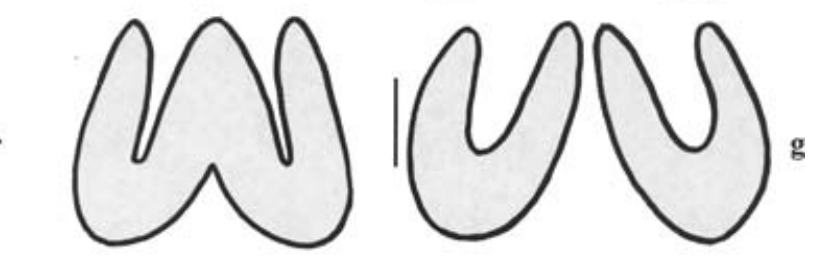

c.
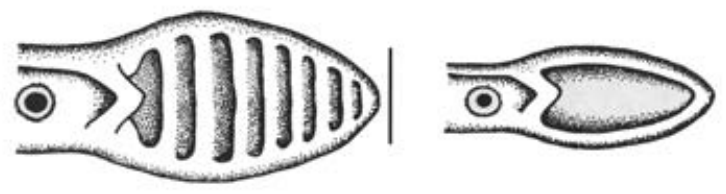

d.
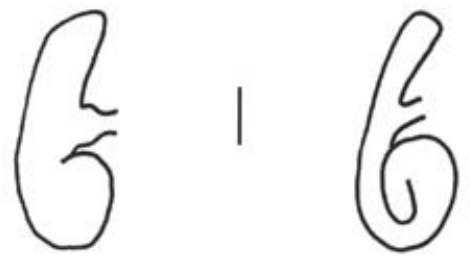

e.
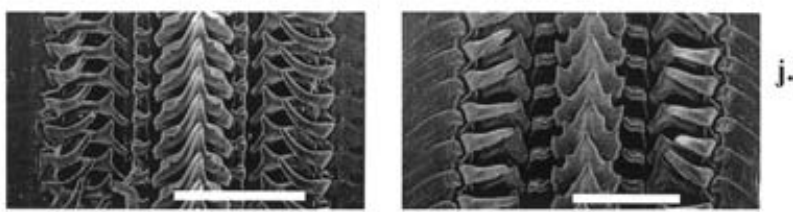

Fig. 3. Comparative anatomy of a-e. Adelieledone polymorpha, NMSZ 2002037.029, ơ, $75 \mathrm{~mm}$ ML and $\mathbf{f}-\mathbf{j}$. Pareledone turqueti, NMSZ 2002037.046, ○َ, 78 mm ML (right).

a, f. Lower beaks, b, g. Funnel organ, c, h. Hectocotylus, d, i. Penis, e, j. Radula.

Scale bars a-d, $f-i=5 \mathrm{~mm}, \mathrm{e}, \mathrm{j}=0.5 \mathrm{~mm}$.

species is from Adélie Land, Antarctica) and Eledone.

Remarks: Members of this genus may be distinguished from Pareledone s.s. by the presence of chromatophores within the connective tissue covering the dorsal surface of the digestive gland (absent in Pareledone), the sharp rostral tip of the lower beak (Fig. 3a) (rounded in Pareledone; Fig. 3f), the presence of transverse ridges in the ligula groove (Fig. 3c) (absent in Pareledone; Fig. 3h), the smaller size of the spermatophores (SpLI 60-80 in Adelieledone, SpLI 100-200 in Pareledone), the uncoiled penis diverticulum (Fig. 3d) (coiled in Pareledone; Fig. 3i), the size of the posterior salivary glands (up to twice the length of the buccal mass in Adelieledone; Fig. 2a, less than the length of the buccal mass in Pareledone; Fig. 2b), the absence of stylets (small cartilaginous stylets are present in Pareledone) and the presence of longitudinal integumentary ridges on the dorsal posterior mantle (Figs 1a \& 4a) (absent in Pareledone).

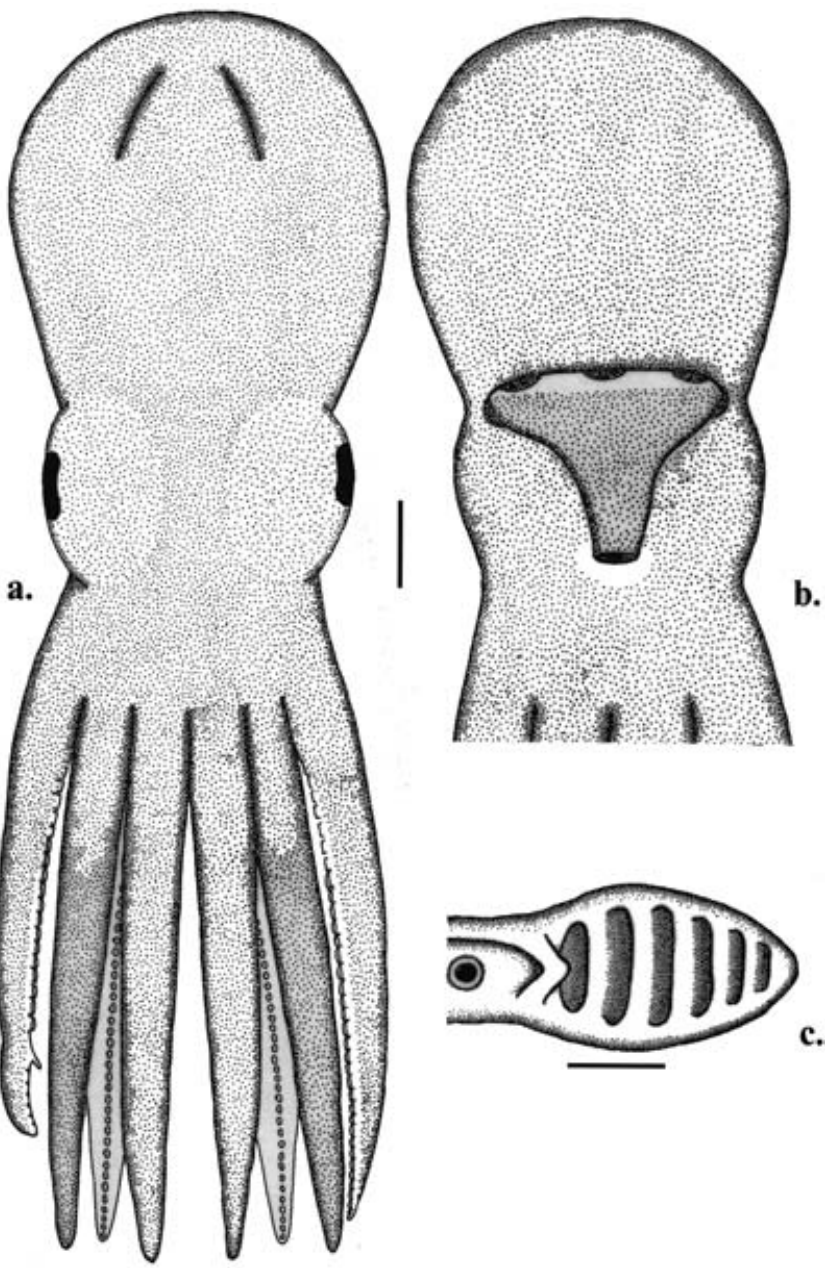

Fig. 4. External anatomy of Adelieledone piatkowski, NMSZ 2000081.053, ơ , $58 \mathrm{~mm}$ ML: a. dorsal view, b. ventral view, c. hectocotylus. Scale bars $\mathrm{a}, \mathrm{b}=10 \mathrm{~mm}, \mathrm{c}=5 \mathrm{~mm}$.

The clear differences in beak shape between Pareledone and Adelieledone will be particularly pertinent to researchers involved in predator/prey studies in the Southern Ocean. Because octopod lower beaks show less morphological variability than teuthid beaks, octopod beaks found in predator stomachs are often identified simply as Octopodidae sp. (for examples see Klages 1996). Pareledone beaks conform to the octopodid norm (Fig. 3f) and are therefore hard to identify from stomach contents, however the beaks of Adelieledone are quite unusual in morphology (Fig. 3a). Because of these differences, Adelieledone specific equations will be required when estimating prey biomass. However the sharp rostral point of the lower beak provides a clear and easy character by which researchers can identify beaks of Adelieledone.

The unusual beak shape of Adelieledone is accompanied by unusually large posterior salivary glands. As suggested by Daly \& Rodhouse (1994), it is likely that Adelieledone is occupying a different trophic niche from Pareledone, but diet studies are needed to confirm this. 
Table I. Adelieledone polymorpha (Robson, 1930). Specimens in the type series.

\begin{tabular}{|c|c|c|c|c|c|c|c|}
\hline $\begin{array}{l}\text { Catalogue } \\
\text { number }\end{array}$ & Station data & $\begin{array}{l}\text { Robson's } \\
\text { identification }\end{array}$ & $\begin{array}{c}\text { Current } \\
\text { identification }\end{array}$ & $\begin{array}{l}\text { Type } \\
\text { status }\end{array}$ & Sex & Maturity & $\begin{array}{c}\mathrm{ML} \\
(\mathrm{mm})\end{array}$ \\
\hline 1951.4 .26 .26 & $\begin{array}{l}\text { Station 42. East Cumberland Bay. } \\
\text { Large otter trawl. } 1 \text { April 1926. 120-204 m. }\end{array}$ & polymorpha & polymorpha & holotype & $\sigma^{x}$ & mature & 52 \\
\hline 1951.4 .26 .27 & $\begin{array}{l}\text { Station } 45.2 .7 \text { miles S } 85^{\circ} \mathrm{E} \text { of Jason Light. } \\
\text { Large otter trawl. } 6 \text { April } 1926.238-270 \mathrm{~m} \text {. }\end{array}$ & oblonga & polymorpha & holotype & $0^{x_{1}}$ & submature & 44 \\
\hline 1951.4.26.28 & $\begin{array}{l}\text { Station 39. East Cumberland Bay. } \\
\text { Large otter trawl. } 25 \text { April 1926. 179-235 m. }\end{array}$ & oblonga & polymorpha & paratype & $\sigma^{x}$ & mature & 47 \\
\hline 1951.4.26.29 & $\begin{array}{l}\text { Station MS 68. East Cumberland Bay. } \\
\text { Large rectangular net. } 2 \text { March 1925. 220-247 m. }\end{array}$ & oblonga & polymorpha & paratype & 우 & mature & 52 \\
\hline 1951.4.26.30 & $\begin{array}{l}\text { Station MS 68. East Cumberland Bay. } \\
\text { Large rectangular net. } 2 \text { March 1925. 220-247 m. }\end{array}$ & oblonga & polymorpha & paratype & $\sigma^{x}$ & submature & 38 \\
\hline 1951.4 .26 .31 & $\begin{array}{l}\text { Station 42. Mouth of Cumberland Bay. } \\
\text { Large otter trawl. } 1 \text { April 1926. 120-204 m. }\end{array}$ & affinis & polymorpha & holotype & $\sigma^{x}$ & mature & 39 \\
\hline 1951.4.26.38-39 & $\begin{array}{l}\text { Station } 148 \text { - off Cape Saunders. } \\
\text { Large otter trawl. } 9 \text { January } 1927.132-148 \text { m. } 54^{\circ} 03^{\prime} \mathrm{S} 36^{\circ} 39^{\prime} \mathrm{W} \text {. }\end{array}$ & $\begin{array}{rr}\text { affinis } \\
\text { V. } & \text { affinis }\end{array}$ & $\begin{array}{l}\text { polymorpha } \\
\text { turqueti }\end{array}$ & $\begin{array}{l}\text { paratype } \\
\text { paratype }\end{array}$ & $\begin{array}{l}\text { q } \\
\text { q }\end{array}$ & $\begin{array}{l}\text { submature } \\
\text { immature }\end{array}$ & $\begin{array}{l}43 \\
28\end{array}$ \\
\hline 1951.4.26.40 & $\begin{array}{l}\text { Station MS 63. East Cumberland Bay. } \\
\text { Small beam trawl. } 24 \text { February } 1925.23 \mathrm{~m} \text {. }\end{array}$ & affinis & turqueti & paratype & $\sigma^{x}$ & mature & 45 \\
\hline 1951.4.26.41-44 & $\begin{array}{l}\text { Station MS 62. Wilson Harbour. } \\
\text { Small beam trawl. } 19 \text { January 1927. 15-45 m. }\end{array}$ & $\begin{array}{l}\text { affinis } \\
\text { affinis } \\
\text { affinis } \\
\text { affinis }\end{array}$ & $\begin{array}{l}\text { polymorpha } \\
\text { polymorpha } \\
\text { polymorpha } \\
\text { turqueti }\end{array}$ & $\begin{array}{l}\text { paratype } \\
\text { paratype } \\
\text { paratype } \\
\text { paratype }\end{array}$ & $\begin{array}{l}0^{x} \\
0^{x} \\
0^{x_{1}} \\
0^{x_{1}}\end{array}$ & $\begin{array}{l}\text { immature } \\
\text { immature } \\
\text { immature } \\
\text { immature }\end{array}$ & $\begin{array}{l}25 \\
13 \\
18 \\
18\end{array}$ \\
\hline
\end{tabular}

${ }^{1}$ originally listed as a female by Robson (1930).

Adelieledone polymorpha (Robson, 1930) new combination (Figs 1, 2a, 3a-e, Tables I-III)

Graneledone polymorpha Robson, 1930: 390, pl 3, fig. 1.

Pareledone polymorpha Robson 1932, 276, pl 5, fig. 1; Daly \& Rodhouse 1994, 165, figs. 3, 7; Piatkowski et al. 1998, 43; Allcock et al. 2001, 833; Allcock \& Piertney 2002, 113, fig. 2 .

Material examined: BMNH 1951.4.26. Type series of Graneledone polymorpha Robson, 1930, see Tables I \& II. [NB. Robson catalogued the material in 1937 as BMNH 1937.1.21 but could not find the specimens at this time. The entire collection, comprising 24 specimens, was reregistered by H. Ricketts on 26 April 1951, hence the new number series BMNH 1951.4.26.]

South Georgia: RRS Discovery: BMNH 20030250, $10^{\pi}$ (13 mm ML), off mouth of Stromness Harbour, Stn 144, 5 January $1927,155-178 \mathrm{~m}, 54^{\circ} 04^{\prime} \mathrm{S}, 36^{\circ} 27^{\prime} \mathrm{W}$. BMNH
20030251, $1 \sigma^{\top}$ (23 mm ML), Stn MS 71, 9 March 1926, 60-110 m. RV John Biscoe: BMNH 1996099, $10^{\top}$ (60 mm ML), Cumberland East Bay, 10 January 1987. BMNH 1996099, 1 우 (58 mm ML), Cumberland East Bay, 27 January 1987, $207 \mathrm{~m}$.

South Orkneys: RRS Discovery: BMNH 20030252, $10^{\pi}$ (26 mm ML), off Signy, Stn 167, 20 February 1927, 244-344 m, 6050'30'S, $46^{\circ} 15^{\prime} \mathrm{W}$.

South Shetlands: RV Polarstern ANT XVII/3: NMSZ 2000081.022, $10^{\top}$ (59 mm ML), 1 우 (54 mm ML), Stn 56/158-1, 26 April 2000, 94-95 m, 6304.7'S, 57³1.6'W. NMSZ 2000081.030, $10^{\top}$ (59 mm ML), 2 우 (41, $62 \mathrm{~mm}$ ML), Stn 56/166-1, 28 April 2000, $666 \mathrm{~m}, 63^{\circ} 02.3^{\prime} \mathrm{S}$, $59^{\circ} 10.4^{\prime} \mathrm{W}$. NMSZ 2000081.035, $10^{\top}$ (49 mm ML), 2 우 (34, $50 \mathrm{~mm}$ ML), Stn 56/173-1, 30 April 2000, 352-279 m, $63^{\circ} 01.2^{\prime} \mathrm{S}, 61^{\circ} 08.7^{\prime} \mathrm{W}$. NMSZ 2000081.041, 1 juvenile. (12 mm ML), Stn 56/175-1, 30 April 2000, 304-305 m, $63^{\circ} 01.0^{\prime} \mathrm{S}, 61^{\circ} 08.8^{\prime} \mathrm{W}$. NMSZ 2000081.043, $10^{\top}(55 \mathrm{~mm}$ 


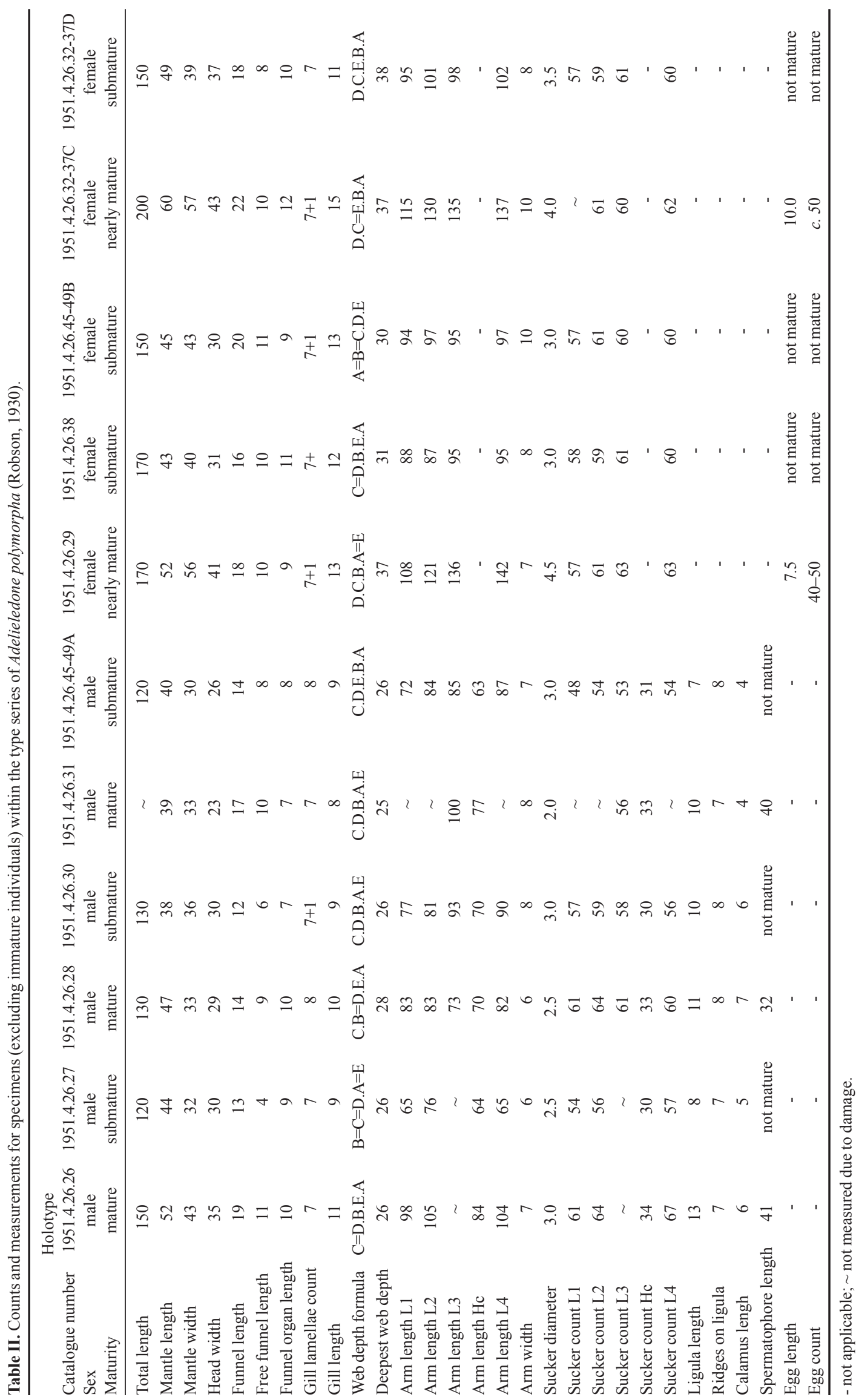

I 
Table III. Comparison of counts and indices for Adelieledone adelieana (from the redescription by Lu \& Stranks 1994) and A. polymorpha (measured from the type series). Indices are given in the format min-meanmax. Most noticeable differences highlighted in bold.

\begin{tabular}{lcc}
\hline & A. adelieana & A. polymorpha \\
\hline Mantle length (ML) & to $\mathbf{5 5} \mathbf{~ m m}$ & to $\mathbf{6 0} \mathbf{~ m m}$ \\
Total length (TL) & to $\mathbf{1 6 0} \mathbf{~ m m}$ & to $\mathbf{2 0 0} \mathbf{~ m m}$ \\
Mantle width index (MWI) & $62.7-76.1-91.1$ & $70.2-86.4-107.7$ \\
Head width index (HWI) & $67.8-77.3-91.6$ & $59.0-69.5-78.9$ \\
Funnel length index (FuLI) & $29.1-34.4-41.3$ & $29.5-36.0-44.4$ \\
Mantle arm index (MAI) & $45.3-58.4-81.5$ & $55.9-68.0-83.5$ \\
Arm length index (ALI) L1 & $\mathbf{1 1 2 . 9 - 1 5 4 . 6 - 2 0 5 . 2}$ & $\mathbf{1 4 7 . 7 - 1 9 0 . 2 - 2 0 8 . 9}$ \\
Arm length index (ALI) L2 & $\mathbf{1 1 5 . 0 - 1 6 1 . 8 - 2 1 6 . 5}$ & $\mathbf{1 7 2 . 7 - 2 0 4 . 8 - 2 3 2 . 7}$ \\
Arm length index (ALI) L3 & $\mathbf{1 1 7 . 6 - 1 6 5 . 0 - 2 1 4 . 2}$ & $\mathbf{1 5 5 . 3 - 2 2 0 . 8 - 2 6 1 . 5}$ \\
Arm length index (ALI) L4 & $\mathbf{1 1 2 . 4 - 1 6 8 . 1 - 2 2 1 . 0}$ & $\mathbf{1 4 7 . 7 - 2 1 2 . 3 - 2 7 3 . 1}$ \\
Arm sucker index (ASI) & $4.3-6.8-10.1$ & $5.1-6.7-8.7$ \\
Opposite arm index (OAI) & $81.2-91.5-98.0$ & $74.1-80.6-95.9$ \\
Hectocotylised arm index (HcAI) & $131.0-156.1-171.0$ & $145.5-165.8-197.4$ \\
Ligula length index (LLI) & $9.7-12.8-15.0$ & $11.1-13.7-15.7$ \\
Calamus length index (CaLI) & $45.1-45.4-46.6$ & $40.0-54.9-63.6$ \\
Hectocotylised sucker count & $\mathbf{2 2 - 2 8}$ & $\mathbf{3 0 - 3 4}$ \\
Opposite arm sucker count & up to 46 & up to 63 \\
Web depth index (WDI) & $21.2-27.1-32.5$ & $24.8-30.0-37.3$ \\
Web formula & $\mathrm{B}=\mathrm{C}=\mathrm{D} . \mathrm{A} . \mathrm{E}$ & $\mathrm{B}=\mathrm{C}=\mathrm{D} . \mathrm{A}=\mathrm{E}$ \\
Gill lamellae count & $\mathbf{6 - 7}$ & $\mathbf{7 - 8}$ \\
Egg length index (EgLI) & $17.3-17.9-18.4$ & $14.4-15.5-16.7$ \\
Spermatophore length index (SpLI) & $58.7-66.9-78.7$ & $68.1-73.5-78.8$ \\
Ligula ridges & approx. 8 & $7-8$ \\
\hline & & \\
\hline & &
\end{tabular}

ML), 2 ㅇ (37, $45 \mathrm{~mm} \mathrm{ML),} \mathrm{Stn} \mathrm{56/177-1,} 1$ May 2000, 200-202 m, 62 ${ }^{\circ} 49.5^{\prime} \mathrm{S}, 60^{\circ} 49.3^{\prime} \mathrm{W}$. NMSZ 2000081.048, 1 우 (70 mm ML), Stn 56/178-2, 2 May 2000, 804-930 m, $61^{\circ} 58.5^{\prime} \mathrm{S}, 60^{\circ} 18.7^{\prime} \mathrm{W}$. NMSZ 2000081.055, 3 우 $(50,51$, 75 mm ML), Stn 56/183-1, 3 May 2000, 200-204 m, $62^{\circ} 06.7^{\prime} \mathrm{S}, 60^{\circ} 21.7^{\prime} \mathrm{W}$. NMSZ 2000081.063, $10^{\top}(58 \mathrm{~mm}$ ML), Stn 56/184-1, 3 May 2000, 338-374 m, 62 $00.9^{\prime} \mathrm{S}$, $60^{\circ} 20.7^{\prime} \mathrm{W} . \quad \mathrm{RV}$ Polarstern ANT XIX/3: NMSZ 2002037.028, $10^{\pi}$ (58 mm ML), Stn 61/047-1, 30 January 2002, $190 \mathrm{~m}, 61^{\circ} 04.2^{\prime} \mathrm{S}, 54^{\circ} 36.6^{\prime} \mathrm{W}$. NMSZ 2002037.029, $50^{\text {ㄱ }}(75,75,74,64,55 \mathrm{~mm} \mathrm{ML}), 5$ 우 $(67,57,50,47,33 \mathrm{~mm}$ ML), Stn 61/048-1, 30 January $2002,343-278 \mathrm{~m}, 61^{\circ} 09.6$ 'S $54^{\circ} 33.6^{\prime} \mathrm{W}$.

Palmer Archipelago: RRS Discovery: BMNH 20030253, 1 ㅇ (29 mm ML), $20^{\pi}$ (17 mm and $14 \mathrm{~mm} \mathrm{ML),} \mathrm{Palmer}$ Archipelago, Neumayr Channel, Stn 187, 18 March 1927, $295 \mathrm{~m}, 6^{\circ} 48^{\prime} 30^{\prime \prime} \mathrm{S}, 63^{\circ} 31^{\prime} 30^{\prime \prime} \mathrm{W}$. BMNH 20030254, 1 우 (16 mm ML), Palmer Archipelago, Anvers Island, Fournier Bay, Stn 186, 16 March 1927, 295 m, 64²5'30"S, 63²'W.

Comparative material examined:

Moschites adelieana Berry, 1917 holotype. AM C40889, Adélie Land, off Mertz Glacier, Mawson Antarctic Expedition, Stn 2, 28 December 1913, 450-549 m, 66 5 55'S, $145^{\circ} 21^{\prime} \mathrm{E}$.

Moschites aurorae Berry, 1917 holotype. AM C40891, off Queen Mary Land, Mawson Antarctic Expedition, Stn 8, 27 January $1914,219 \mathrm{~m}, 66^{\circ} 08^{\prime} \mathrm{S}, 9^{\circ} 17^{\prime} \mathrm{E}$.
Eledone turqueti Joubin, 1905 holotype. MNHN 5.7.1089, Île Wandel, Charcot Antarctic Expedition.

Eledone charcoti Joubin, 1905 holotype. MNHN 5.7.1095. Île Wandel, Charcot Antarctic Expedition.

Pareledone framensis Lu \& Stranks, 1994 paratype. NMV F65667, Prydz Bay, RV Aurora Australis, Stn AA91-100, 28 February $1991,145-150 \mathrm{~m}, 67^{\circ} 27.35^{\prime} \mathrm{S}, 68^{\circ} 50.34^{\prime} \mathrm{E}$.

Pareledone prydzensis Lu \& Stranks, 1994 paratype. NMV F65625, Prydz Bay, RV Aurora australis, Stn AA91-89 (2), 24 February $1991,526-532 \mathrm{~m}, 66^{\circ} 47^{\prime} \mathrm{S}, 72^{\circ} 36^{\prime} \mathrm{W}$.

Moschites harrissoni Berry, 1917 holotype. AM C40892, Queen Mary Land, off Shackleton Glacier, Mawson Antarctic Expedition, Stn 10, 29 January 1914, 494-595 m, $65^{\circ} 06^{\prime} \mathrm{S}, 96^{\circ} 13^{\prime} \mathrm{E}$.

Pareledone turqueti (Joubin, 1905). NMSZ 2002037.046, Antarctic Peninsula, RV Polarstern ANT XIX/3, Stn 61/048-1, 30 January 2002, 343-278 m, 6109.6'S, $54^{\circ} 33.6^{\prime} \mathrm{W}$.

Diagnosis: Rostral tip of lower beak sharp (Fig. 3a). Ligula long (LLI 11-16) with well-marked groove crossed by 7-8 transverse ridges (Fig. 3c). Arms short to medium (mean ALI L1 190, L2 205, L3 221, L4 212). Hectocotylized arm with 30-34 suckers. Gills with 7-8 lamellae per demibranch. Widely scattered papillae on dorsal surface (Fig. 1a). Ventral surface smooth and creamy white (Fig. 1b).

Description: (Based on mature and submature animals in Robson's type series, Table II.) Animals small to medium sized (ML to $60 \mathrm{~mm}$; TL to $200 \mathrm{~mm}$ ). Mantle spherical to ovoid (MWI 70.2-86.4-107.7), head narrower than mantle (HWI 59.0-69.5-78.9). Funnel small (FuLI 29.5-36.0-44.4), gently tapered; funnel organ W-shaped (Fig. 3b). Arms short to medium length (MAI 55.9-68.0-83.5). Arm lengths subequal, arm order usually $3=4.2 .1$ (ALI L1 147.7-190.2208.9; L2 172.7-204.8-232.7; L3 155.3-220.8-261.5; L4 147.7-212.3-273.1). Suckers uniserial, small (ASI 5.1-6.78.7), without sucker enlargement. Third right arm of males hectocotylized, shorter than opposite number (OAI 74.180.6-95.9). Ligula large (LLI 11.1-13.7-15.7); ligula groove long, well marked and deep, with 7-8 transverse ridges (Fig. 3c). Calamus distinct and large (CaLI 40.0-54.9-63.6). Hectocotylized arm with 30-34 suckers, opposite arm with up to 63 suckers. Web of medium depth (WDI 24.8-30.037.3), web formula approximately $B=C=D . A=E$. Ink sac present. Chromatophores present within the connective tissue covering the dorsal surface of digestive gland. Posterior salivary glands large, up to twice the length of the buccal mass (Fig. 2a). Gills with 7-8 lamellae per demibranch. Rostral tip of lower beak sharp (Fig. 3a). Mature ovarian eggs large $(>10 \mathrm{~mm})$. Penis diverticulum uncoiled (Fig. 3d). Spermatophores medium sized (SpLI 68.1-73.5-78.8) and slender. Males mature above $45 \mathrm{~mm}$ 
Table IV. Counts and measurements for specimens of Adelieledone piatkowski. Specimens for which no repository is given were not retained.

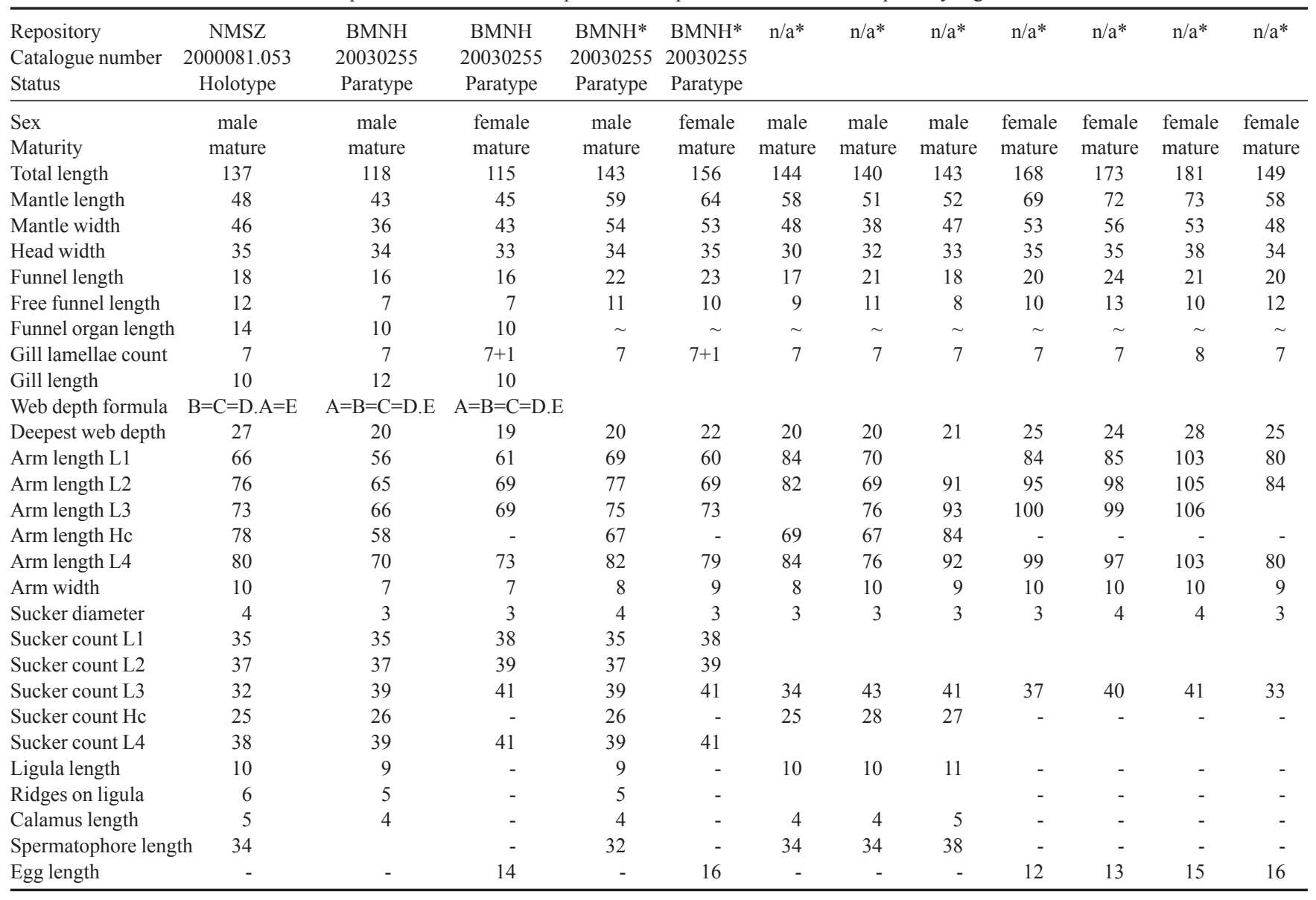

- not applicable; $\sim$ not measurable on fresh tissue; $*$ measurements made prior to fixation in formalin.

ML, females mature above $60 \mathrm{~mm}$ ML.

The skin is often loose and gelatinous prior to fixation. The skin sculpture consists of fine, widely scattered papillae on the dorsal surface. Two short, longitudinal integumentary ridges are present on the mid-dorsal posterior mantle. A ventrolateral integumentary ridge is also present (Fig. 1a). The ventral surface is smooth and creamy white in colouration with a few scattered chromatophores laterally (Fig. 1b). Chromatophores are densely packed on the dorsal surface, which varies (even on a single animal) from brown to green to blue. White markings are usually present on the dorsal brachial crown (often in the form of an inverted $\mathrm{V}$ ) and smaller areas of leucophores may be present on the dorsal mantle and arms, although these display no obvious regular pattern (Fig. 1a).

Type locality: Southern Ocean, South Georgia, mouth of Cumberland Bay. RRS Discovery, 120-204 m depth, 1 April 1926, large otter trawl.

Distribution: Occurs around South Georgia in depths from $116 \mathrm{~m}$ to $364 \mathrm{~m}$. Further comment on distribution is made in the remarks section.

Etymology: From the Greek polu (much) and morphe (form), because of the variability of mantle shape seen in this species.

Remarks: The identification of seven specimens of Pareledone turqueti within the type series of Adelieledone polymorpha (Table I), significantly reduces the variability of form seen. Examples of Robson's subspecies affinis and oblonga still remain within the series, but the series includes neither mature nor submature males of the form affinis as these specimens were re-identified as $P$. turqueti. We found no validity for the separation of the species into two forms during our re-examination of the type series, nor during extensive fishing around South Georgia. Adelieledone polymorpha has gelatinous skin and is probably prone to distortion effects during preservation. This, combined with the inclusion of incorrectly identified material in the type series, probably led to the unwarranted subdivision of A. polymorpha into two morphological forms. We conclude that the forms affinis and oblonga are not valid.

Specimens from the Antarctic Peninsula do show some morphological variation compared to those from South Georgia. Specimens have been recorded with larger mantle lengths (up to $75 \mathrm{~mm}$ ). The arms are slightly shorter (mean ALI L1 162, L2 173, L3 180, L4 179) and there is slight 
variation in the hectocotylized arm sucker count (26-32). Gill lamellae counts and other counts and measurements appear however to be consistent with those from specimens around South Georgia and we assume that the two forms are conspecific.

\section{Adelieledone adelieana (Berry, 1917) new combination} (Table III)

Moschites adelieana Berry, 1917, 17, text figs 10-13, pl. 11, fig. 5, pl. 12 figs $6-8$.

Pareledone adelieana Robson 1932, 278; Lu \& Stranks 1994, 222, figs 1, 9a-d.

Pareledone umitakae Taki 1961, 308, text figs 9-16, pl. 3.

Material examined: AM C40889. Holotype of Moschites adelieana Berry, 1917. 1 female (25 mm ML). Adélie Land, off Mertz Glacier, Mawson Antarctic Expedition, Stn 2, 28 December 1913, 450-549 m, 66 $55^{\circ} \mathrm{S}, 145^{\circ} 21^{\prime} \mathrm{E}$.

Comparative material examined: NMSZ 2000081.003, $10^{\pi}$ (51 mm ML), Weddell Sea, RV Polarstern ANT XVII/3,

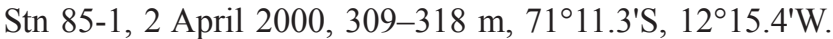
NMSZ 2000081.010, $10^{\top}$ (80 mm ML), 1 우 (30 mm ML), Weddell Sea, RV Polarstern ANT XVII/3, Stn 102-1, 3 April 2000, 312-323 m, 71 ${ }^{\circ} 11.9^{\prime} \mathrm{S}, 12^{\circ} 21.7^{\prime} \mathrm{W}$. NMSZ 2000081.018, 1 우 (48 mm ML), Weddell Sea, RV Polarstern ANT XVII/3, Stn 136-1, 10 April 2000, 260-271 m, $70^{\circ} 50.2^{\prime} \mathrm{S}, 10^{\circ} 35.4^{\prime} \mathrm{W}$.

Diagnosis: Rostral tip of lower beak sharp. Ligula long (LLI 10-15) with well-marked groove crossed by approximately eight transverse ridges. Arms short (mean ALI L1 155, L2 162, L3 165, L4 168). Hectocotylized arm with 22-28 suckers. Gills with 6-7 lamellae per demibranch. Widely scattered papillae on dorsal surface. Ventral surface smooth and creamy white.

Description: See Lu \& Stranks 1994.

Type locality: Adélie Land, off Mertz Glacier, eastern Antarctica $66^{\circ} 55^{\prime} \mathrm{S}, 145^{\circ} 21^{\prime} \mathrm{E}$ in $450-549 \mathrm{~m}$ depth.

Distribution: On the continental shelf and slope of Antarctica from approximately $30^{\circ} \mathrm{E}$ to $90^{\circ} \mathrm{E}$ in depths from $139-680 \mathrm{~m}$. Further comment on distribution is made in the remarks section.

Etymology: Although not explicitly stated by Berry, the name derives from the type locality, Adélie Land.

Remarks: This species from East Antarctica, previously treated under the name Pareledone adelieana Berry, 1917, is morphologically similar to Adelieledone polymorpha. It is herein placed in the genus Adelieledone. Adelieledone adelieana differs from $A$. polymorpha in colour (A. adelieana is purple-pink to purple-grey dorsally; Lu \& Stranks 1994) and in size at maturity (A. adelieana grows to a total length of $160 \mathrm{~mm}$, males mature at $40 \mathrm{~mm}$ ML, females at $45 \mathrm{~mm} \mathrm{ML;} \mathrm{Lu} \mathrm{\&} \mathrm{Stranks} \mathrm{1994).} \mathrm{The} \mathrm{original}$ description of $A$. adelieana was based on one female specimen (TL $110 \mathrm{~mm}$ ) that was caught together with two juvenile specimens (TL 18 and $16 \mathrm{~mm}$ ) from $66^{\circ} 55^{\prime} \mathrm{S}$, $145^{\circ} 21^{\prime} \mathrm{E}$ in $288-300$ fathoms. The holotype was reexamined as part of this study but the specimen is distorted, several of the arms are damaged and the radula is missing. A fuller description of the species was provided Lu \& Stranks (1994) following extensive fishing in the Southern Ocean between $50^{\circ} \mathrm{E}$ and $75^{\circ} \mathrm{E}$. This redescription, based on 10 immature and five mature males, and ten submature, one mature and one spent female, included the standard counts and indices commonly used in octopod taxonomy. Comparison of these measures (Table III) with those derived from the type series of $A$. polymorpha shows that the species are separated by relatively few characters. A. polymorpha appears to have relatively longer arms (mean ALI L1 190.2 in A. polymorpha versus 154.6 in A. adelieana) which can bear a greater number of suckers (up to 63 in A. polymorpha versus up to 46 in A. adelieana), more suckers on the hectocotylized arm (30-34 in A. polymorpha versus $22-28$ in $A$. adelieana) and marginally more gill leaflets ( $7-8$ in $A$. polymorpha versus 6-7 in A. adelieana).

It is not clear whether material from the Weddell Sea (see comparative material examined) is conspecific with A. adelieana. Specimens have slightly shorter arms (mean ALI L1 140, L2 153, L3 158, L4 156), the same number of gill lamellae as $A$. adelieana (6-7) and a slightly higher hectocotylized arm sucker count (25-30). The type localities of $A$. polymorpha and A. adelieana are widely separated and it is likely that these species are valid, especially considering the small differences over which genetic divergence is shown to occur in Antarctic octopuses (Allcock et al. 1997). However, there is clearly a continuum of morphological forms around Antarctica and the specific status of these forms can probably only be resolved using molecular techniques.

Pareledone umitakae Taki, 1961 is morphologically similar to $A$. adelieana and is considered a junior synonym of this species (Lu \& Stranks 1994). The type of this species is not available for study. It was presumed extant in a private collection, but it appears that the material has been either mislaid or destroyed (I. Gleadall, personal communication, 2003) so further consideration of this nominal species is not possible.

\section{Adelieledone piatkowski sp. nov.}

(Fig. 4, Table IV)

Pareledone sp. A. Allcock \& Piertney 2001, 131, fig. 2.

Pareledone cf polymorpha type 1. Piatkowski et al. 1998, 43.

Material examined: NMSZ 2000081.053, holotype, $10^{x}$ (58 $\mathrm{mm} \mathrm{ML}$ ), Antarctic Peninsula, ANT XVII/3, RV Polarstern, Stn 178-2, Commercial bottom trawl, 2 May 
$2000, \quad 804-930 \mathrm{~m}, \quad 61^{\circ} 58.5^{\prime} \mathrm{S}, \quad 60^{\circ} 18.7^{\prime} \mathrm{W}-61^{\circ} 57.3^{\prime} \mathrm{S}$, $60^{\circ} 16.7^{\prime} \mathrm{W}$. BMNH unreg., paratypes, $10^{\top}$ (59 mm ML), 1 우 (64 mm ML), Antarctic Peninsula, ANT XIV/2, RV Polarstern, Stn 42/045, 27 November 1996, 807 m, $61^{\circ} 42^{\prime} \mathrm{S}, 59^{\circ} 10^{\prime} \mathrm{W}$. BMNH unreg., paratypes, 2 우 $(21 \mathrm{~mm}$ and $22 \mathrm{~mm} \mathrm{ML}$ ), Antarctic Peninsula, ANT XIV/2, RV Polarstern, Stn 42/044, 27 November 1996, 583 m, $61^{\circ} 37^{\prime} \mathrm{S}, 58^{\circ} 46^{\prime} \mathrm{W}$.

In addition, the following material was examined fresh, but not retained, during expedition ANT XIV/2 of RV Polarstern to the Antarctic Peninsula in 1996, see Table IV: $30^{\top}$ (58 mm, $51 \mathrm{~mm}$ and $\left.52 \mathrm{~mm} \mathrm{ML}\right)$, Stn 42/045, 27 November 1996, $807 \mathrm{~m}, 61^{\circ} 42^{\prime} \mathrm{S} 59^{\circ} 10^{\prime} \mathrm{W} .70^{\top}(64 \mathrm{~mm}$, $68 \mathrm{~mm}, 60 \mathrm{~mm}, 60 \mathrm{~mm}, 63 \mathrm{~mm}, 57 \mathrm{~mm}$ and $57 \mathrm{~mm} \mathrm{ML}$ ), 9 ㅇ $(68 \mathrm{~mm}, 69 \mathrm{~mm}, 73 \mathrm{~mm}, 62 \mathrm{~mm}, 58 \mathrm{~mm}, 60 \mathrm{~mm}$, $53 \mathrm{~mm}, 52 \mathrm{~mm}$ and $58 \mathrm{~mm} \mathrm{ML}$ ), Stn 42/048, 28 November 1996, $785 \mathrm{~m}, 61^{\circ} 35^{\prime} \mathrm{S} 58^{\circ} 45^{\prime} \mathrm{W}$. 1우 (72 mm ML), Stn 42/145, 19 December 1996, 1514 m, 62 ${ }^{\circ} 15^{\prime} \mathrm{S} 56^{\circ} 57^{\prime} \mathrm{W}$.

Diagnosis: (Based on the type material listed above; additional variation may be inferred from the counts and measurements on unpreserved material given in Table IV.) Beak small; rostral tip of lower beak sharp. Ligula groove long, well-marked and deep with 5-6 transverse ridges (Fig. 4c). Arms short. Hectocotylized arm with 25-26 suckers. Gills with 7-8 lamellae per demibranch. Integument loose and smooth apparently without papillae, although these may become apparent after the animal is fixed. Dorsal and ventral surfaces covered in dense purplish grey chromatophores (Fig. 4a \& b).

Description: (Based on the type material listed above; additional variation may be inferred from the counts and measurements on unpreserved material given in Table IV.)

Animals small to medium sized (ML to $50 \mathrm{~mm}$; TL to $140 \mathrm{~mm}$ ). Mantle spherical to ovoid (MWI 83.7-91.7-95.8), head narrower than mantle (HWI 72.9-75.1-79.1). Funnel small (FuLI 35.6-36.8-37.5), gently tapered; funnel organ W-shaped. Arms short to medium length (MAI 60.0-61.061.6). Arm lengths subequal, arm order usually $4.3=2.1$ (ALI L1 130.2-134.4-137.5; L2 151.2-154.3-158.3; L3 152.1-153.0-153.5; L4 162.2-163.9-166.7). Suckers uniserial, small (ASI 6.7-7.3-8.3), without sucker enlargement. Third right arm of males hectocotylized, on average slightly shorter than opposite number (OAI 87.997.4-106.8). Ligula large (LLI 11.1-13.7-15.7); ligula groove long, well marked and deep, with 5-6 transverse ridges. Calamus distinct and large (CaLI 44.4-47.2-50.0). Hectocotylized arm with 25-26 suckers, opposite arm with up to 41 suckers. Web of medium depth (WDI 26.0-29.433.8), web formula approximately $\mathrm{A}=\mathrm{B}=\mathrm{C}=\mathrm{D}$.E. Ink sac reduced. Chromatophores within the connective tissue covering the dorsal surface of digestive gland. Posterior salivary glands large (up to twice the length of the buccal mass). Gills with 7-8 lamellae per demibranch. Rostral tip of lower beak sharp. Mature ovarian eggs large (> $14 \mathrm{~mm}$ ).
Spermatophores medium sized (SpLI approximately 70) and slender. Males and females appear to be mature above a mantle length of $45 \mathrm{~mm}$.

The integument is loose and smooth apparently without papillae, although these may become apparent after the animal is fixed. Two short longitudinal ridges are present on the mid-dorsal posterior mantle (Fig. 4a). A ventrolateral integumentary ridge may be raised but is more apparent in fixed than in fresh specimens. All surfaces are completely covered in purplish grey chromatophores, except for two areas around the funnel that remain white (Fig 4b).

Type locality: Antarctica, Antarctic Peninsula, 61 ${ }^{\circ} 58.5^{\prime} \mathrm{S}$ $60^{\circ} 18.7^{\prime} \mathrm{W}-61^{\circ} 57.3^{\prime} \mathrm{S} 60^{\circ} 16.7^{\prime} \mathrm{W}$. RV Polarstern, 804-930 m depth, 2 May 2000, commercial bottom trawl.

Distribution: Antarctica: Antarctic Peninsula in depths from $612-1510 \mathrm{~m}$.

Etymology: Named after Dr Uwe Piatkowski, Institut fur Meereskunde, Germany.

Remarks: This species may be distinguished from Adelieledone polymorpha and Adelieledone adelieana by the presence of densely packed chromatophores on the ventral surface and by the apparent absence of papillae (although see above). The latter is possibly a reflection of the poor condition of these animals when removed from deep trawls (A. polymorpha is often taken alive from shallow trawls) as scattered papillae may be discernible in fixed material. The hectocotylus of $A$. piatkowski has fewer transverse ridges (Fig. 4c) than the hectocotylus of A. polymorpha (Fig. 3c). In all members of the genus, the ink sac appears to be set deep within the digestive gland (Fig. 2a); in Adelieledone piatkowski, however, it appears also to be reduced in size. This is perhaps an adaptation to the increased depths that this species inhabits. The remainder of the anatomy is extremely similar to that of A. polymorpha and is therefore not illustrated.

\section{Pareledone Robson, 1932} (Figs $3 \mathrm{f}-\mathrm{j}$ )

Diagnosis: Benthic octopodids, ML to $120 \mathrm{~mm}$; mantle saccular, without fins; eight arms lacking cirri; arms with small (ASI 5-12) uniserial suckers, third right arm of male hectocotylized with end of arm clearly differentiated into ligula and calamus, ligula medium to large (LLI 6-13), ligula groove long, well marked and shallow without transverse ridges (Fig. 3h), arm tips not otherwise modified; web depth medium (WDI 20-50); funnel organ VV-shaped (Fig. 3g); gills well developed, with 6-11 lamellae; ink sac present; crop well developed, posterior salivary glands medium size, length of posterior salivary glands approximately equal to length of buccal mass (Fig. 2b); cartilaginous stylets present but extremely delicate; diverticulum of penis coiled (Fig. 3i), spermatophores long (SpLI 100-200) and slender; rostral tip of lower beak 
rounded (Fig. 3f); radula with nine elements (Fig. 3j), rachidian with large central cusp, rachidian may have other cusps.

Type species: Eledone charcoti Joubin, 1905. By subsequent designation.

Included species: Eledone charcoti Joubin, 1905, Eledone turqueti Joubin, 1905, Moschites harrissoni Berry, 1917, Moschites aurorae Berry, 1917, Moschites antarcticus Thiele, 1920, Pareledone prydzensis Lu \& Stranks, 1994, Pareledone framensis, Lu \& Stranks, 1994.

Etymology: Although not explicitly stated by Robson, the name derives from the combination of the Greek word Para meaning 'beside' with Eledone.

Remarks: The diagnosis of Pareledone is now simplified. The ligula groove is now restricted to a long shallow groove without transverse ridges. The funnel is restricted to VVshaped, although caution must be exercized with this character. The funnel organ often does not preserve well and two closely adjacent Vs can resemble a W shape; for this reason we have not included the funnel organ in the diagnostic features for Adelieledone and Pareledone as it might lead to confusion. The size of the salivary glands is now restricted to a much smaller range, with salivary glands being slightly shorter in length to the length of the buccal mass. The range of length of spermatophores is also restricted. It is likely that the coiled penis diverticulum seen in Pareledone is to accommodate the somewhat longer spermatophores. The beak shape is limited to the normal octopodid form where the lower beak has a rounded tip.

The valid species of Pareledone still fall into two categories: those with smooth skin (P. turqueti, P. harrissoni and $P$. antarctica) and those with papillated skin (P. charcoti, $P$. aurorae, P. prydzensis and P. framensis). It is unclear whether these constitute two separate clades and this issue may need to be address using molecular markers.

\section{Acknowledgements}

The Alfred Wegener Institute provided sea-time aboard RV Polarstern. Wolf Arntz, Gerhard Kattner, Karl-Hermann Kock and Uwe Piatkowski helped make this possible. Fishing around South Georgia was made possible through a ground fish survey funded by the South Georgia Government and organized by Graham Parkes (Marine Research Assessment Group) and Inigo Everson (British Antarctic Survey), aboard MV Cordella. Mike Vecchione, Uwe Piatkowski, Silke Steimer and Heather Daly gave advice and assistance at sea. Fred Naggs and Amelia MacLellan (BMNH), Melanie McKenzie and Tim Stranks (MV), Virginie Héros and Philippe Bouchet (MNHN), I. Loch and Holly Barton (AM) and Sankurie Pye (NMSZ) provided access to specimens. Electron microscopy was done under a Sharing Museums Skills Millenium Award to
ALA. Alex Ball and Chris Jones (BMNH) assisted with the microscopy. Ian Gleadall provided the information about Taki's personal collection. Mark Norman and Janet Voight critically reviewed and helped improve the manuscript. This work was begun under a NERC CASE award to ALA and continued at the National Museums of Scotland.

\section{References}

Allcock, A.L. \& Piertney, S.B. 2002. Evolutionary relationships of Southern Ocean Octopodidae (Cephalopoda: Octopoda) and a new diagnosis of Pareledone. Marine Biology, 140, 129-135.

Allcock, A.L., Brierley, A.S., Thorpe, J.P. \& Rodhouse, P.G.K. 1997. Restricted geneflow and evolutionary divergence between geographically separated populations of the Antarctic octopus Pareledone turqueti. Marine Biology, 129, 97-102.

Allcock, A.L., Piatkowski, U., Rodhouse, P.G.K. \& Thorpe, J.P. 2001. A study on octopodids from the eastern Weddell Sea, Antarctica. Polar Biology, 24, 832-838.

BERRY, S.S. 1917. Cephalopoda. Australian Antarctic Expedition Scientific Reports, Series C, 4(2), 1-38.

Carlini, D.B., Young, R.E. \& Vecchione, M. 2001. A molecular phylogeny of the Octopoda (Mollusca: Cephalopoda) evaluated in the light of morphological evidence. Molecular Phylogenetics and Evolution, 21, 388-397.

Daly, H.I. \& Rodhouse, P.G. 1994. Comparative morphology of two sympatric Pareledone species from South Georgia. Antarctic Science, $\mathbf{6}$, 163-169.

KLages, N.T.W. 1996. Cephalopods as prey. II. Seals. Transactions of the Royal Society of London, B351, 1045-1052.

LIPINSKI, M. \& WoyciechowsKi, M. 1981. Cephalopods in the food of Weddell seals from the Admiralty Bay (King George Island, South Shetland Islands). Polish Polar Research, 2, 163-167.

Lu, C.C. \& Stranks, T.N. 1994. Synopsis of Pareledone and Megaleledone species, with description of two new species from East Antarctica (Cephalopoda: Octopodidae). Memoirs of the National Museum of Victoria, 54, 221-242.

Ogden, R., Allcock, A.L., Watts, P.C. \& Thorpe, J.P. 1998. The usefulness of beak morphology as a taxonomic character in Southern Ocean Octopodidae. South African Journal of Marine Science, 20, $29-36$.

Piatkowski, U., Allcock, L., Hevia, M., Steimer, S. \& Vecchione, M. 1998. Cephalopod ecology. In Kattner, G., ed. The expedition ANTARKTIS XIV/2 of RV Polarstern in 1996/1997. Berichte zur Polarforschung, 274, 41-47.

Piatkowski, U., Allcock, L. \& Vecchione, M. In press. Cephalopod diversity and ecology. Berichte zur Polarforschung.

Robson, G.C. 1930. Cephalopoda. I. Octopoda. Discovery Reports, 2, 371-402.

RoBson, G.C. 1932. A monograph of the recent Cephalopoda based on the collections in the British Museum (Natural History). Part II. The Octopoda (excluding the Octopodinae). London: British Museum, $359 \mathrm{pp}$.

Rodhouse, P.G., Arnbom, T.R., Fedak, M.A., Yeatman, J. \& Murray, A.W.A. 1992. Cephalopod prey of the southern elephant seal, Mirounga leonina L. Canadian Journal of Zoology, 70, 1007-1015.

Roper, C.F.E. \& Voss, G.L. 1983. Guidelines for taxonomic descriptions of cephalopod species. Memoirs of the National Museum of Victoria, 44, 48-63.

TAKI, I. 1961. On two new eledonid octopods from the Antarctic Sea. Journal of the Faculty of Fisheries and Animal Husbandry, Hiroshima University, 3, 297-316.

VoIGHT, J.R. 1993. A cladistic reassessment of octopodid classification. Malacologia, 35, 343-349. 